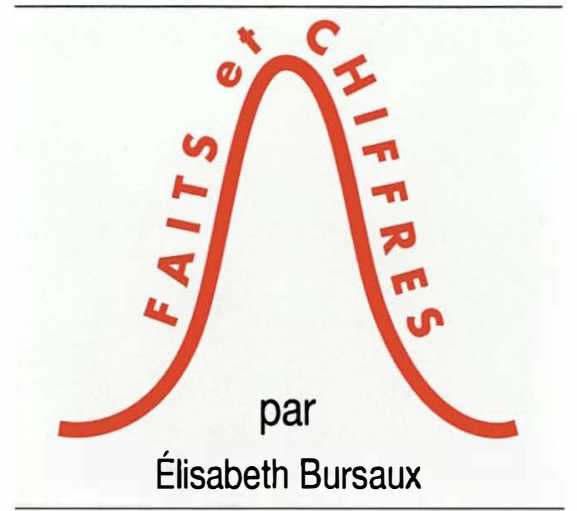

méderine/sriences 1995;11:1037-8

\title{
Inégalités familiales et inégalités sociales de santé
}

$\mathbf{N}$ ous avons rapporté dans ces colonnes les inégalités régionales en France devant la mort $\left(\mathrm{m} / \mathrm{s} n^{\circ} 5\right.$, vol. $\left.10, p .583\right)$, dues en partie aux disparités de recours aux soins $\left(\mathrm{m} / \mathrm{s} n^{\circ} 5\right.$, vol. 10, p. 716). C'est d'un autre type d'inégalité et de ses conséquences sur la santé qu'il s'agit ici: l'influence des problèmes familiaux pendant l'enfance sur les maladies à l'âge adulte. Ce sujet fait l'objet d'une étude de Georges Menahem, au CREDES (Centre de recherche, d'étude et documentation en économie de la santé). Il montre que les problèmes affectifs durables vécus avant 18 ans sont associés à une plus grande fréquence des troubles de santé à l'âge adulte. Un individu sur trois s'est trouvé concerné, soit par un grand manque d'affection, soit par le conflit de ses parents ou leur absence prolongée, soit par la maladie grave de l'un d'entre eux. Ces persomnes ayant vécu de graves difficultés familiales avant 18 ans déclarent $43 \%$ de plus de maladies à l'âge adulte que celles qui n'en ont pas connues. Ces résultats ressortent de l'enquête INSEE «Études des conditions de vie 1986-1987 " analysant les déclarations de maladies de 13150 individus [1] (figure 1).

En revanche, l'expérience précoce du décès d'un parent, ou de leur séparation est beaucoup moins délétère, et le nombre de maladies déclarées par les personnes ayant vécu ces chocs est à peine supérieur à celui des individus n'ayant subi ni problème affectif durable, ni mort, ni séparation.

Ces résultats sont confirmés par trois enquêtes statistiques de l'INSEE (par indices à âge et sexe constants), et par des analyses qualitatives conduites à partir d'une enquête approfondie par récits de vie [2]. Les personnes éprouvées dans leur enfance témoigneraient de moins grandes dispositions à faire attention à leurs problèmes de santé, et cela d'autant moins qu'elles appartiendraient à des catégories sociales défavorisées. L'héritage familial se traduirait par une plus grande propension à prendre des risques dans sa santé et dans son mode de vie, conduisant à des accidents répétés, à des difficultés d'argent et des maladies : par exemple, les individus ayant déclaré avoir connu la mésentente de leurs parents dans leur enfance fument et boivent deux fois plus que ceux ayant eu une enfance sans heurt affectif.

Mais les inégalités sociales mettent là aussi leur marque : les problèmes affectifs durables de l'enfance se traduisent davantage dans les catégories socio-professionnelles d'ouvriers et d'employés par des attitudes imprévoyantes que dans la catégorie des cadres (figure 2) 91-103.
- Menahem C. Problemes de len fance, C.REDES, 1994

. Menahem (;, Martin S. Quand l'enfance 
Figure 1. Variations de déclarations de maladies par rapport à la moyenne selon la catégorie sociale (A) et selon les problèmes affectifs connus avant 18 ans (B). En ordonnées: le supplément par rapport à la moyenne (0) du nombre total de maladies déclarées à l'âge adulte. A. Les individus de la catégorie socio-professionnelle des ouvriers ont déclaré en moyenne $5 \%$ de maladies de plus que la moyenne de l'échantillon, soit $22 \%$ de plus que ceux de la catégorie cadres, pour des groupes dont les proportions selon l'âge et le sexe sont comparables. B. Les individus ayant répondu avoir connu un grand manque $d^{\prime}$ affection dans leur jeunesse ont eu, en moyenne, $49 \%$ de plus de maladies que la moyenne de l'échantillon, et $62 \%$ de plus que ceux n'ayant connu aucun problème affectif durable. II faut noter que l'expérience précoce du décès de l'un des parents ou de leur séparation n'entraîne pas de préjudice de santé à l'âge adulte. 13150 personnes ont participé à l'enquête; les nombres se rapportent au nombre d'individus dans chaque groupe: 1 : n'ayant eu ni choc affectif ni problème durable dans la jeunesse; 2 : ayant subi des chocs affectifs mais sans problème durable; 3: ayant subi des problèmes affectifs durables.

Source : Enquête INSEE "Études des conditions de vie 1986-1987".

Figure 2. Nombre moyen de déclarations de maladies selon les problèmes de la jeunesse dans quatre catégories socio-professionnelles. En ordonnées : indice du nombre de maladies déclarées à l'âge adulte, à âge et sexe comparables; 100 : moyenne nationale. En abscisse, chaque catégorie socio-professionnelle est subdivisée en quatre groupes selon les antécédents affectifs dans la jeunesse. Les troubles de santé sont bien moins fréquents dans la catégorie des cadres que dans les autres catégories lorsque les adultes concernés ont connu des problèmes familiaux dans leur jeunesse.

Source : Enquête INSEE "Études des
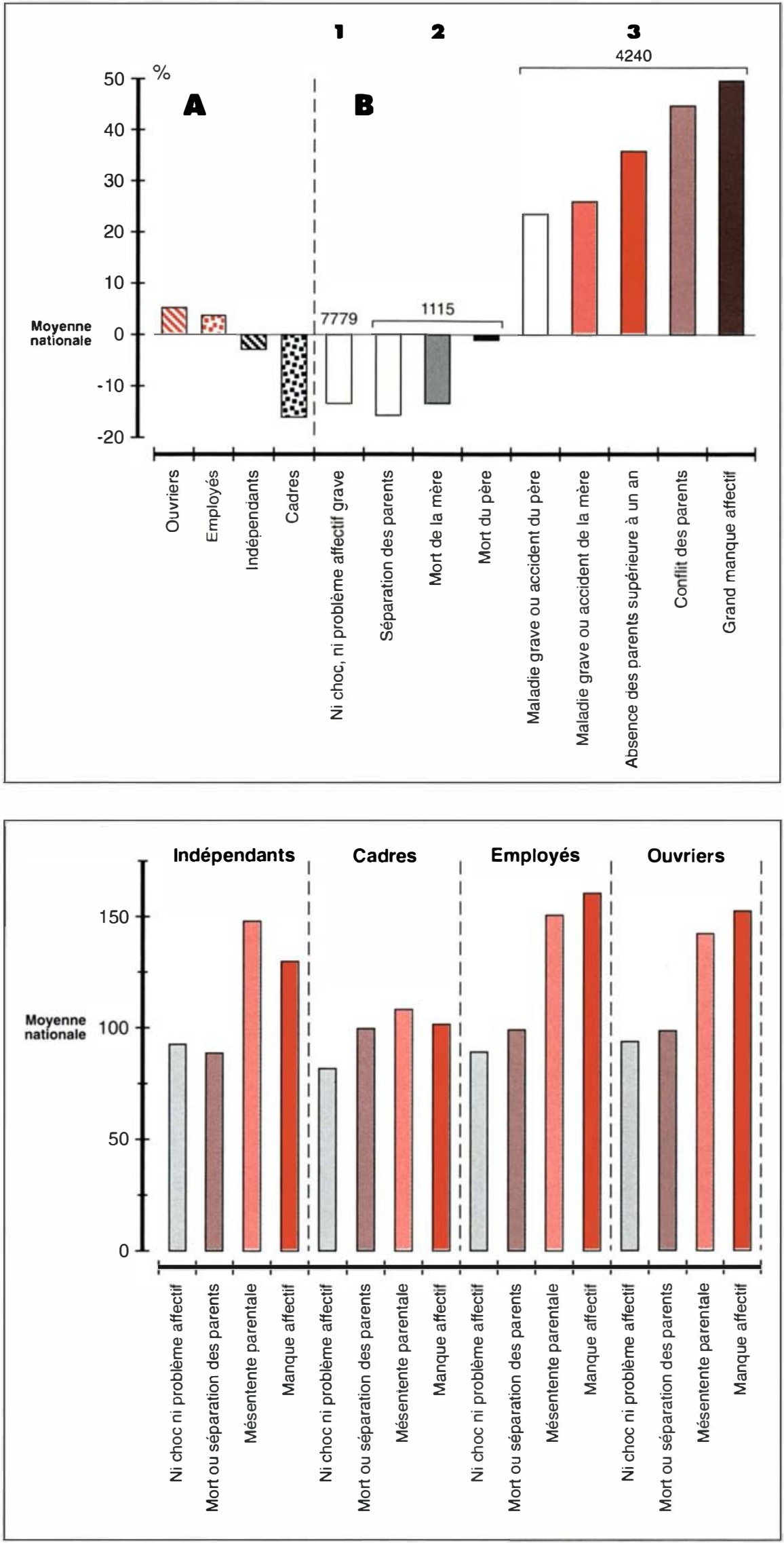